
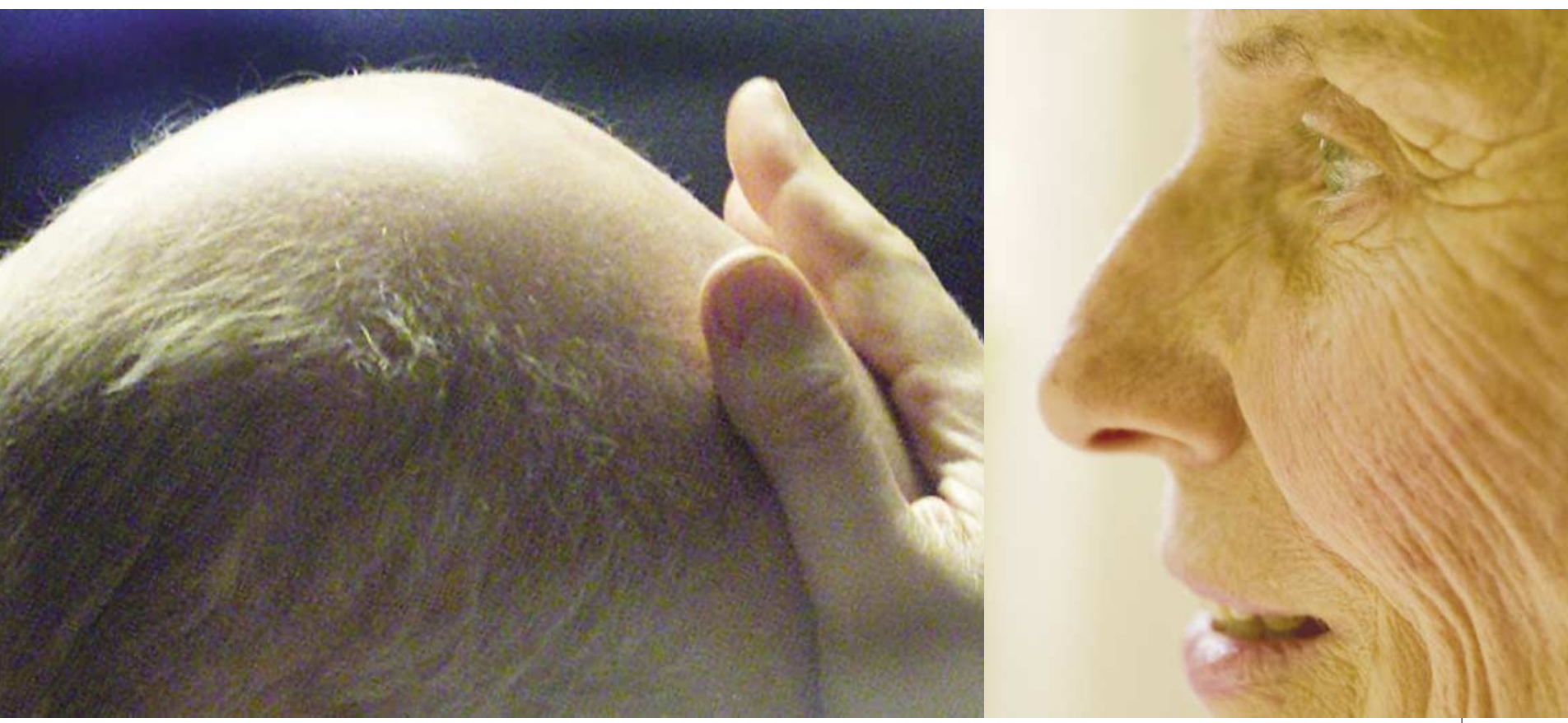

\title{
In the eye of the beholder
}

\author{
Does the pharmaceutical industry's future lie at the boundary between \\ drugs and cosmetics? Or is the prospect of effective 'cosmeceuticals' a \\ beauty myth? Helen Pearson investigates.
}

n 1991, dermatologist Alistair Carruthers made a surprise announcement to his colleagues. "We're going to use the most deadly agent known to humanity for the treatment of wrinkles," he told a meeting of the American Society for Dermatologic Surgery in Orlando, Florida.

That agent - a paralysing protein extracted from the bacteria that cause botulism — was already being marketed as a drug called Botox, used to freeze the overactive muscles that cause crossed eyes. By persuading healthy people to try it off-label, Carruthers and his ophthalmologist wife Jean, at the University of British Columbia in Vancouver, Canada, had shown that it also immobilizes the muscles that furrow the brow. It wasn't until April 2002 that this treatment was formally approved by the US Food and Drug Administration (FDA). But by then, lunchtime Botox 'face-lifts' were already de rigueur in certain social circles.

Botox is not the only agent to have strayed across the blurred line between drugs and cosmetics. Over the past decade, a handful of drugs have found uses in flattening wrinkles, preventing baldness or shedding unwanted hair. Given the demand from a society obsessed by beauty, one might expect pharmaceutical and cosmetics companies to be falling over themselves to create a new generation of vanity drugs - or 'cosmeceuticals', as they are known.

In truth, the action is less than intense in large part because drug firms believe that the big bucks still lie in the treatment of disease. Although US cosmeceutical sales reached $\$ 3.4$ billion in 2002, according to the Freedonia Group, a business research company in Cleveland, Ohio, this is only a tiny fraction of the \$185.2-billion US pharmaceutical market. Even if sales of cosmeceuticals double over the next decade, as the Freedonia Group expects, that's unlikely to change the views of the big drug firms.

\section{Face facts}

The pseudo-scientific reputation of cosmetics, fear of liability should the treatment go wrong, as well as technical obstacles, are also dissuading drug companies from entering the field. But some experts predict that the firms' attitudes could change as their parched product pipelines make them increasingly desperate for commercial success. And if one company hits upon a genuine cosmeceutical blockbuster - say, a drug with no side effects that sprouts hair in all balding men industry executives might soon be fighting to join the fray. "Their attention span could change in a flash," predicts Robert Partridge, director of communications at Dermik Lab- oratories in Berwyn, Pennsylvania, part of Aventis Dermatologicals.

Most cosmetics firms, meanwhile, have little incentive to enter the regulatory morass of the drug business, given that they can make healthy profits from their existing range of creams and lotions. According to the FDA's definition, drugs are agents used in the diagnosis, cure, mitigation, treatment or prevention of disease, or which are intended to affect the structure or function of the body. Cosmetics, on the other hand, don't require extensive testing and FDA approval before they hit the market, because they simply alter our appearance. Cosmeceuticals can be viewed as drugs that affect our appearance, or cosmetics that change the structure or function of our bodies.

The first agent to fall into this category was all-trans retinoic acid, the biologically active form of vitamin A, which is now known to boost the collagen content of the skin. In the early 1980s, when Albert Kligman of the University of Pennsylvania in Philadelphia was testing all-trans retinoic acid as a treatment for acne in women, he found that it smoothed wrinkles caused by exposure to the sun. The drug was subsequently repackaged under the brand name Renova and approved by the FDA as an anti-ageing skin treatment. Low doses of all-trans retinoic acid or its precursor 


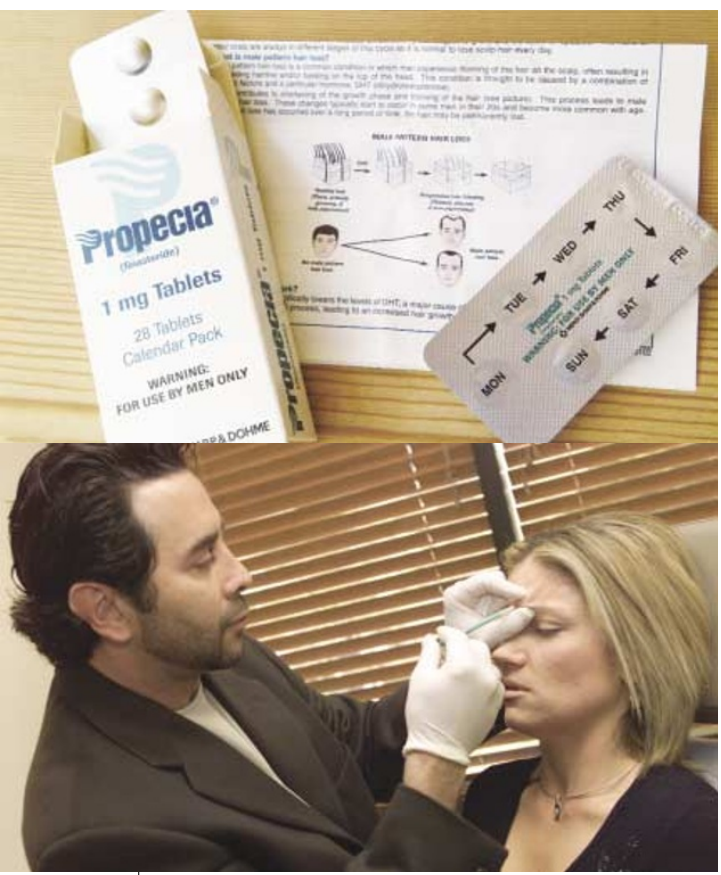

retinol now find their way into numerous over-the-counter face creams.

Most of the other well known cosmeceuticals are similarly drugs whose unexpected side effects have earned them a place in the beauty parlour. Propecia and Rogaine, for example, both of which stall hair loss, were developed to treat benign prostate tumours and high blood pressure, respectively. A recent entrant to the club is Vaniqa, the first prescription drug for removing unwanted hair. It is a topically applied version of a drug that was originally developed to treat African sleeping sickness.

\section{Age concern}

Gauging pharmaceutical companies' current interest in cosmeceuticals is difficult, because most are tight-lipped about their business strategy and research programmes. But drug giant Pfizer admits that it is actively researching new prescription compounds for cosmetic purposes. Last year, it absorbed a company called Anaderm, based in Ann Arbor, Michigan, which it had bankrolled since 1996. Anaderm is working on drugs to tackle five common cosmetic concerns: age spots, oily skin, hair loss, excess hair and sun damage. Its head of clinical exploration, Arthur Bertolino, says that two candidate drugs will go into clinical testing this year but he refuses to reveal any further details.

Pfizer's open interest in cosmeceuticals seems to be the exception, rather than the rule. Merck, which markets Propecia, regards this product as an outlier, and says that it is continuing to concentrate on diseases such as diabetes. Industry observers say that this makes sense: not only are cosmeceuticals likely to make only a fraction of the sum that can be earned by a conventional block-
Youth culture: aspects of ageing such as baldness and wrinkles are now being tackled by treatments such as Propecia (left) and Botox injections, which blur the line between drugs and cosmetics.

buster drug, the potential liabilities are also huge. Patients are normally willing to accept a small risk of experiencing side effects in treating a disease. But someone taking a drug for its cosmetic effects is likely to hold companies liable if side effects crop up. "The risk-benefit ratio is awful,” says George Annas, a bioethicist at Boston University School of Public Health.

On top of this, drug companies are keen to project the image of a serious industry conquering disease, rather than one indulging in frippery. "They want to be associated with real science," says Richard Dixey, chief executive of the drug-discovery company Phytopharm, based in Godmanchester, near Cambridge, UK.

Drugs to treat obesity, on the other hand, are of interest to almost every pharmaceutical giant. Although not usually lumped together with cosmeceuticals, they are an example of the grey line between drugs that treat disease and those used for cosmetic purposes. The two leading anti-obesity prescription drugs, Hoffmann-La Roche's Xenical and Abbott Laboratories' Meridia, are only licensed to treat morbidly obese patients. But obesity researcher Stephen Bloom of Imperial College, London, says that the drugs inevitably find illicit sales to slimmers. "It's kind of self-evident," he says.

Roche and Abbott are adamant that their anti-obesity drugs are not intended for cosmetic use. Indeed, drug companies would gain nothing by aiming anti-obesity drugs at the over-the-counter slimming market any such application would be turned down by the FDA and other national regulators because of the potential for abuse by people with eating disorders.

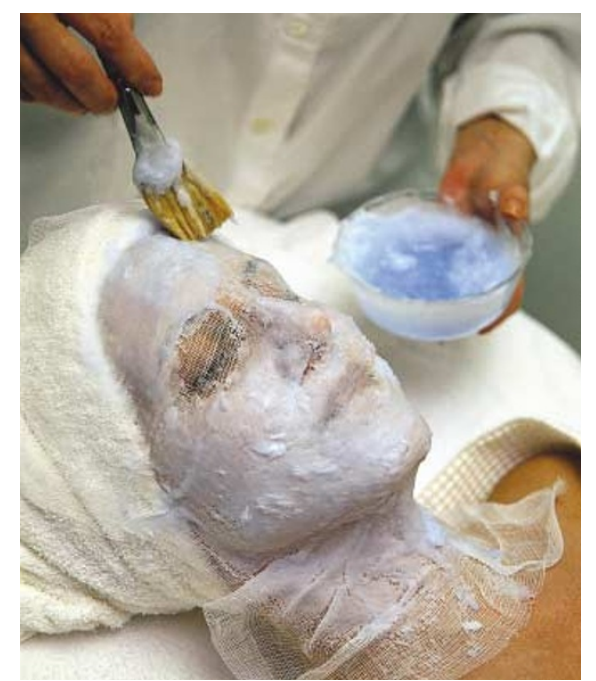

Despite rising demand, drug firms so far seem reluctant to pitch into the cosmetics market.
In the cosmetics industry, meanwhile, only the largest companies can afford the expensive research and clinical trials required to bring a new drug onto the prescription market. Cosmetics giant L'Oréal, for example, teamed up with the food and drinks conglomerate Nestlé in 1981 to create a dermatology-research company called Galderma, headquartered in Lausanne, Switzerland. The company is seeking new drugs to pep up ageing skin or tackle balding, says its vice-president for corporate marketing and business development, Gabriel Villada. He also declines to reveal any details.

\section{Smooth operators}

Many cosmetics firms remain wary about products that have biological activity. Nevertheless, some are exploiting the blurry definition of cosmeceuticals to bring drug-like products to market without attracting the attention of drug regulators. In the United States, if a company finds a new molecule, puts it in a pot and claims that it alters the structure and function of the skin, it must undergo years of expensive clinical trials. But if the marketing claims fudge around the cream's biological activity, it can hurdle straight into the stores. Hence the carefully worded scientific claims featured in many cosmetics adverts. "We don't want to have our products taken off the shelf," says Meagan McLellan, president of cosmetics firm Cellex-C International in Toronto, Canada.

In any case, cosmetics companies are wary of slipping potent ingredients into their creams in case they cause skin irritation. And financially, cosmetics firms seem to get by perfectly well without resorting to drug-like compounds. "There's so much money made already," says Chris Griffiths, a dermatologist at the University of Manchester, UK.

Although both pharmaceutical and cosmetics companies have business reasons to spurn cosmeceuticals, scientific obstacles also remain. The identification of hairrestoring potions, for example, has been hindered by the lack of a laboratory test bed - a scalp-in-a-dish, if you like - on which candidate molecules can be screened, says Angela Christiano, who studies the genetics of hair growth at Columbia University in New York.

But the saggy, shaggy or balding should not lose heart entirely. Entrepreneurial biotechnology companies, and pharmaceutical subsidiaries such as Anaderm, are quietly chipping away at the cosmeceutical coalface. So far, most of the drug giants are keeping their distance. "They'll only move into a new area if it has blockbuster potential," says Tom Dooley, chief executive of the dermatologyresearch company IntegriDerm in Birmingham, Alabama. But if IntegriDerm or another start-up firm does find the cosmeceutical equivalent of Prozac or Viagra, the big companies' attitude may change overnight. Helen Pearson works in Nature's syndication news team. 